\title{
La evolución del discurso de la gestión empresarial a través de la literatura gerencial. Hacia la constitución del sujeto neoliberal*
}

\author{
The Evolution of the Discourse on Business Management through the Management Literature. Towards the \\ Constitution of a Neoliberal Subject
}

A evolução do discurso da gestão empresarial através da literatura gerencial. Para a constituição do sujeito neoliberal

\author{
Maria Medina-Vicent ${ }^{\text {a }}$ \\ Universitat Jaume I, España \\ medinam@uji.es \\ ORCID: http://orcid.org/0000-0002-2716-6786
}

DOI: https://doi.org/10.11144/Javeriana.syp38-75.edge

Fecha de recepción: 18 Mayo 2018

Fecha de aprobación: 30 Noviembre 2018

Fecha de publicación: 15 Noviembre 2019

\section{Resumen:}

En el presente artículo se pretende reflexionar en torno a los principales rasgos de la literatura gerencial, poniendo especial énfasis en su capacidad de adaptarse a los cambios históricos que se dan en el sistema económico para amoldar las subjetividades contemporáneas a las necesidades de dicho sistema. Con el fin de conseguir este objetivo, el documento explotará el potencial del storytelling de estas obras a la hora de establecer prescripciones de comportamiento a sus lectores/as, y se enfocará en las argumentaciones morales que priman en el discurso gerencial actual, y en el proceso de mercantilización y formación del nuevo sujeto neoliberal al que contribuyen.

Palabras clave: literatura gerencial, capitalismo sólido, sociedad líquida, neoliberalismo, sujeto neoliberal.

\section{Abstract:}

This article intends to reflect on the main traits of the management literature by putting special emphasis on the ability to adapt oneself to the historic changes in the economic system, so that the contemporary subjectivities may fit the needs in this system. To attain this aim, this document explores the storytelling potential in these works regarding the way they provide behavior prescriptions to the readers, and focuses on the moral arguments prevailing in the management discourse nowadays as well as the process of commodification and formation of a new neoliberal subject it contributes to.

Keywords: management literature, solid capitalism, liquid society, neoliberalism, neoliberal subject.

\section{Resumo:}

O artigo objetiva fazer revisão de estudos sobre o consumo de práticas culturais em jovens de algumas universidades latinoamericanas. Entende-se como prática cultural as atividades referentes a mídia escrita e audiovisual, museus, exposições, esportes virtuais, tecnologias de informação e comunicação. Foram analisadas 52 pesquisas de artigos, revistas indexadas, teses e relatórios, realizados entre os anos 2001 e 2018, nos quais houve avanços e lacunas nesta temática. As análises desses estudos permitem concluir que o consumo cultural dos jovens universitários mudou em consequência da globalização, o avanço das tecnologias da informação e comunicação e a influencia das novas indústrias culturais.

Palavras-chave: literatura gerencial, capitalismo sólido, sociedade líquida, neoliberalismo, sujeito neoliberal.

\section{Introducción}

La literatura gerencial refleja el espíritu económico de cada época histórica. Por esta razón, adentrarnos en su análisis permite conocer el discurso de la gestión empresarial de cada tiempo. De hecho, dichas obras reflejan y difunden las modas de gestión más populares en el mundo del management entre la clase directiva mundial

Notas de autor:

\footnotetext{
a Autora de correspondencia. Correo electrónico: medinam@uji.es
} 
y el público general (Clark, 2004; Collins, 2000). Es decir, inciden en la configuración de las imágenes sobre la gestión empresarial en el imaginario colectivo (Fernández Rodríguez y Medina-Vicent, 2017). Así pues, los valores presentes en estas influyen en la conformación de los discursos sobre la dirección empresarial y en la formación de sus futuros/as profesionales, por lo que se trata de espacios en los que se crean, procesan y divulgan las ideas del management siguiendo el modelo de producción cultural (Hirsch, 1972). Sin embargo, también cabe destacar que sus principios alcanzan la lógica vital de la ciudadanía, es decir que influyen en las subjetividades contemporáneas de los sujetos, no solamente dentro del ámbito empresarial, sino también en su sentido más social.

Por tanto, se puede afirmar que dicha literatura influye en la formación de los/as profesionales de la dirección empresarial, lo que hace que se tiendan a incorporar una serie de valores y lógicas por encima de otras. De este modo, y si se tiene en cuenta que dicha literatura refleja las modas de gestión de cada tiempo, seremos conscientes de que dichos valores van a variar en función de los intereses de legitimación de cada sistema económico. Desde nuestro punto de vista, consideramos que el riesgo de esta realidad reside en el consumo y la recepción acrítica de las ideas expuestas en estos libros por parte de sus lectores/as, tal y como señala el autor Andrzej Huczynski (1993, 2011). El acriticismo con el que se tratan los temas de estas obras (el liderazgo, la gestión del cambio, el aprendizaje continuo, el rendimiento, etc.) (Boyett y Boyett, 1999), y con el que se reciben e incorporan, nos hace plantearnos los riesgos morales que tiene para la práctica profesional del/la manager atender a esta lógica argumental y dejar de lado el análisis estructural de las desigualdades sociales, así como los problemas y tensiones que lo caracterizan. En adición, nos hace plantearnos cómo este influye en la constitución de la subjetividad neoliberal actual.

Un ejemplo de esta cuestión la encontramos en la obra Filosofía y Sociedad, en la cual Mario Bunge (2008) entrevista al conocido estudioso del mundo de los negocios, Henry Mintzberg. Este último autor advierte de la acogida acrítica que tienen las ideas de los gurús dentro de las escuelas de negocios y de las empresas, y cita el caso de Enron como un ejemplo de los resultados negativos que puede tener la puesta en práctica de ciertas modas del management. Citando el estrepitoso fracaso de Enron en la aplicación de ciertas técnicas de gestión (Bastidas, 2007), Mintzberg subraya la necesidad de acoger críticamente las modas de gestión y de reflexionar sobre los efectos de su aplicación; idea que apoya James Hoopes (2003), estudioso de esta realidad. Por esta razón, debemos preguntarnos críticamente acerca de sus ideas y su papel en el desarrollo empresarial a lo largo de diferentes épocas, algo que haremos en este trabajo.

Así pues, la estructura de sentido que vertebra el presente trabajo se configura de las siguientes partes. A continuación, trataremos de reflexionar en torno a una de las funciones principales de la literatura gerencial, esto es, la legitimación de la posición de la élite directiva en los entornos laborales y sociales. Más tarde, nos centraremos en el potencial del storytelling de estas obras a la hora de conectar con el público lector y establecer prescripciones de comportamiento, en los patrones de conducta que condicionan los modos de gestionar una empresa, y en los modos en que gestionamos nuestros propios proyectos vitales. Seguidamente, abordaremos la literatura gerencial como un objeto de estudio vivo que se encuentra en constante desarrollo y se adapta a los cambios que acontecen en el sistema económico, social, tecnológico y político que nos rodea. Nos centraremos, pues, en la evolución del discurso gerencial, situando el foco de atención en el momento en que se pasa del capitalismo sólido al líquido. Por último, este será el puente que hará posible abordar el cambio de estas prescripciones a lo largo de las décadas, en función de las necesidades del sistema económico y de trabajo de cada momento, y que permitirá establecer una conclusión sobre las argumentaciones morales que priman en el discurso gerencial actual y el proceso de mercantilización y formación del nuevo sujeto neoliberal. 


\section{La legitimación de la élite directiva a través de la literatura gerencial}

Parte de la influencia de la literatura gerencial en la actuación de las empresas recae en la naturaleza divulgativa de este tipo de obras, rasgo que les permite llegar a un mayor público, al contrario de los conocimientos académicos, cuya acogida resulta más lenta y problemática. Por esta razón, la literatura gerencial se ha configurado como fuente de conocimiento alternativo a la literatura académica (Álvarez y Mazza, 2000; Gómez Álvarez y Sánchez Barrios, 2009). En este sentido, el autor David Riesman (1998) sugiere que el incremento de la influencia de dicha literatura de carácter más popular se debe, en parte, a la colonización de las instituciones educativas y de la producción científica por parte de los valores de consumo. Esto provoca que en muchas ocasiones las ideas lanzadas por parte de la literatura gerencial se vuelvan de uso común, incluso antes de formar parte de la formación académica (Engwall, 2012; Sahlin-Andersson y Engwall, 2002) o de la gestión empresarial práctica.

Sin embargo, las razones del éxito de esta literatura entre los/as managers sobrepasa su carácter divulgativo y ha sido ampliamente discutida, lo que ha permitido encontrar diferentes puntos de explicación (Clark y Salaman, 1998, pp. 140-143). En primer lugar, se encuentra la vía psicológica, es decir, la tesis de que en un mundo tan variante e impredecible como el de la gestión empresarial, la literatura de la gestión otorga a los/ as managers la sensación de tener el control de la situación (Watson, 2004), más que realmente tenerlo. En segundo lugar, otros arguyen que su éxito se debe a que el estilo comunicativo con el que son redactadas dichas obras es de fácil lectura y absorción, algo que parece sincronizar con el estilo de los/as managers (Mintzberg, 2005). En tercer lugar, se han señalado las formas de aprender de los profesionales como explicación para su receptividad hacia las ideas de los gurús, algunos señalan que es su aprendizaje orientado a la acción lo que les predispone a escuchar dichas ideas (Honey y Mumford, 1982). En cuarto lugar, podría deberse a que los gurús empresariales hacen gala de las cualidades que todo manager desearía poder tener, como, por ejemplo, el carisma o la elocuencia (Vail, 1991), por lo que la lectura de las obras se convertiría en una actividad con gran carácter aspiracional. Por último, también se ha tenido en cuenta que más que producir prácticas de sentido en lo que se refiere a la gestión empresarial, se reconstruyen los significados que ya se hallan en dicho ámbito, y se racionalizan en un aspecto concreto de su significado (Willmott, 1993). En esta medida, contribuyen a divulgar ciertas concepciones sociales de la empresa, y su gestión entre la clase directiva y el propio entorno social.

Pero quizás, la razón principal del éxito de esta literatura entre la clase directiva es que permite justificar la posición de superioridad de la élite managerial, configurada claramente como una élite social en las sociedades occidentales actuales (Abrahamson, 1991). Al fin y al cabo, las prescripciones que se pueden encontrar en la literatura managerial están directamente relacionadas con la profesión de los/as managers y su capacidad para hacer valer su poder. $Y$ es que dichos profesionales se encargarán de traducir las premisas de gestión de la literatura a la práctica (Gowler y Legge, 1991), por tanto, deberán reproducir el rol de la gerencia y los significados que le son socialmente asignados. Por tanto, no es de extrañar que dicha literatura sirva para legitimar la ideología managerial y, con ella, la posición de poder de la élite directiva. En este sentido, cabe preguntarse cómo afecta esta realidad los valores y comportamientos que se difunden mediante este tipo de literatura. Es decir, ¿contribuye esta legitimación del poder de la élite empresarial a transformar las subjetividades contemporáneas hacia un modelo de sujeto neoliberal basado en la lógica de la gestión de uno/ a mismo/a? Esta es una de las preguntas que se tratarán de resolver en este trabajo.

\section{Storytelling y prescripción}

En su objetivo de difusión de las modas de gestión y la posición privilegiada de la élite directiva, la literatura managerial adquiere dos rasgos principales: la posición de superioridad moral desde la que se escriben los 
textos y el carácter prescriptivo de sus contenidos. En primer lugar, el rasgo central que permite concebir dicha literatura como un reflejo de la posición social de los cargos directivos, es que dentro de los propios textos se habla desde la posición de la dirección. Se trata de una posición privilegiada y caracterizada por la superioridad de mando con respecto al resto de trabajadores/as. El autor Brad Jackson (2001), en su obra Management Gurus and Management Fashions, señala que los gurús de la gestión tienden a divulgar una visión de la organización con la que la dirección pueda identificarse. Con esta operación se pretende que el público lector, formado por los cuadros directivos de las empresas, se sienta parte del relato, y se configure como protagonista del mismo en un entorno real.

Desde siempre, el storytelling ha sido clave en el mundo empresarial; sin embargo, en los últimos años la necesidad de crear una cultura corporativa resulta más apremiante. La progresiva precarización del trabajo aumenta la necesidad de conseguir la adhesión de todo el equipo humano a la misión de la empresa. En esta misma línea, el autor Christian Salmon (2008) identifica el desarrollo del storytelling del management producido a partir de mediados de los noventa, como una técnica para el cambio radical de la gestión empresarial tradicional que pasa por la inclusión del storytelling en la comunicación interna de la empresa como instrumento de motivación de los/as trabajadores/as. Dicho neomanagement se nutre de las historias de sus trabajadores/as para crear una narración colectiva, que, según su posición, tiene una función legitimadora del status del/la trabajador/a dentro de la estructura de la empresa.

Por otro lado, Barbara Czarniawska (1997a, 1997b) también analiza el papel de la narrativa en los estudios de la organización, y señala su creciente relevancia en dicho campo a la hora de divulgar conocimiento. La narración ofrece la oportunidad de enseñar a estudiantes las prácticas del mundo organizacional de un modo más efectivo que la escritura científica, ya que les resulta más fácil extraer la lección de aplicación al ámbito organizacional. Por medio de las historias, metáforas y narraciones se construyen parte de los significados compartidos en las organizaciones, sin que lo importante, al contrario de lo que ocurre con la escritura lógicocientífica, sea el discernimiento entre los hechos y la verdad. Así, el storytelling configura el espacio más pertinente para negociar los significados presentes en el mundo empresarial (Czarniawska, 1995), y así lo refleja la literatura gerencial, que se nutre de la narración para hacerse con la complicidad de su público lector.

Sin embargo, la cuestión de la relación entre lector/a y autor/a es más compleja que la identificación que se pretende conseguir mediante el storytelling, y ha sido ya discutida. A continuación, se tratará de abordar brevemente dicha discusión. Si bien dicha relación se puede concebir desde una perspectiva más unidireccional, en la cual la primera figura sería un sujeto pasivo que queda a la espera de las ideas del gurú, y que adquiere sus obras en el intento por desarrollar una ventaja competitiva sobre el resto de la clase directiva (Thomas, 2003), también se puede comprender desde una visión bidireccional en la cual ambas partes obtienen beneficios, tal y como señalan Clark y Salaman (1998). Mientras que en la primera idea se concibe a los/as managers como consumidores/as de ideas, en la segunda visión se puede hablar de una relación de construcción mutua de los significados. Sin embargo, en ambos casos, las obras del management pretenden que se implementen ideas de gestión en la misma práctica.

En segundo lugar, está el carácter prescriptivo de sus contenidos, que está ligado a su carácter divulgativo. Su gran éxito de divulgación se evidencia a partir de los grandes beneficios económicos que genera la industria de la literatura gerencial (Clark y Salaman, 1998; Jackson, 2001), así como del éxito de los gurús de la gestión a lo largo de sus trayectorias profesionales (Jackson, 2001). Así pues, ante la disolución de las grandes teorías económicas convertidas en ideas, conceptos y premisas vulgarizadas, la gestión empresarial se aborda de una forma muy simplista al adquirir un gran carácter prescriptivo que aporta recetas prácticas para el management de éxito. Se podría señalar que con la compra se espera el acceso a una serie de claves que van a dar resultados en la actividad profesional, como indica Huczynski (2006). En su intención de ofrecer pautas para el éxito empresarial a los cuadros directivos, existe una clara tendencia a definir qué es lo correcto y lo incorrecto en la tarea de la gestión organizacional. 
Cuando se habla del discurso gerencial divulgado mediante la literatura del management, se estaría hablando de un discurso moralizante que pretende guiar los comportamientos de los/as directivos/as hacia el éxito empresarial (Alonso y Fernández Rodríguez, 2013). Las prescripciones se configuran en una dicotomía correcto-incorrecto en un sentido técnico-instrumental; es decir, acciones acertadas o equivocadas entre las diferentes alternativas. Mientras que las decisiones correctas mejorarán la productividad y aumentarán la motivación del equipo de trabajo, las incorrectas provocarán lo contrario (Fernández Rodríguez, 2007). Dicha construcción dicotómica y prescriptiva nos otorga el escenario adecuado para abordar la evolución de la literatura gerencial y los cambios que se dan en sus prescripciones en el paso del capitalismo sólido al capitalismo líquido, en el que nos encontramos actualmente.

Y es que los discursos gerenciales no son estáticos ni permanentes, sino más bien al contrario. Se trata de discursos que se atienen a los cambios sociales, de cualquier otro modo perderían su vigencia rápidamente. Un ejemplo paradigmático es el afamado gurú, Peter Drucker, quien por su larga vida vivió diferentes épocas, y sus escritos reflejan las tendencias de diversas modas de gestión. Así, un análisis de la obra de Drucker "permite comprobar cómo el discurso refleja las transformaciones del sistema productivo, escenificando la transición del modelo de producción en serie al de producción flexible: una metáfora de las cadenas es sustituida por otra metáfora de las redes" (Fernández Rodríguez, 2008, p. 214). En el siguiente apartado se intentarán rastrear las principales etapas de la evolución de las modas de gestión reflejadas en la literatura gerencial de las últimas décadas, para luego establecer una conclusión sobre los valores preeminentes en la época actual y sobre qué tipo de sujeto se pretende construir.

En referencia, en la actualidad esta literatura "se muestra como uno de los principales lugares de inscripción del espíritu del capitalismo" (Boltanski y Chiapello, 2002, p. 98). Dicha realidad permite deducir que la literatura gerencial reproduce los valores asociados a ese nuevo espíritu, "influenciando la forma en que se organiza el trabajo dentro de las organizaciones" (Alonso y Fernández Rodríguez, 2013, p. 42). Por tanto, resulta vital identificar los valores sobre los que se sustenta el discurso gerencial hoy en día, para ver qué modelo de individuo tratan de configurar.

\section{La evolución del discurso gerencial: del capitalismo sólido al líquido}

Como se comentó en el apartado anterior, el discurso gerencial se encuentra a merced de la evolución económica y social, así como de los cambios argumentales de la propia ideología capitalista y los nuevos desarrollos del ámbito tecnológico. Esta realidad empuja al surgimiento de diferentes modas de gestión que pueden sucederse unas a otras, o incluso coincidir en el tiempo en su intento por ofrecer a sus lectores/as la posibilidad de conseguir el éxito empresarial. Por esta razón, se propone realizar una breve reflexión sobre el paso del capitalismo sólido al líquido.

El debate entre capitalismo sólido y capitalismo líquido es una de las discusiones filosóficas protagonistas de nuestro tiempo. La acepción de capitalismo sólido o pesado se encuentra en Das Kapital, Kritik derpolitischen Ökonomie, de Marx, y se refiere al momento en que el capitalismo giraba en torno al concepto de producción. Se trataba de un sistema intensivo de mano de obra en que el valor añadido y el beneficio se obtenían básicamente del diferencial entre el pago del trabajo, convertido en mercancía, y el precio obtenido de la producción fruto de este trabajo. Este modelo se mantuvo hasta después de la Segunda Guerra Mundial, y a finales de los sesenta se inicia el paso al capitalismo líquido, en el cual la producción da paso a la productividad, introduciendo como pieza fundamental del mecanismo productivo la eficiencia y el valor de los procesos. Con el concepto de capitalismo líquido, Bauman $(2001,2003)$ intenta vislumbrar el paso del capitalismo industrial al financiero. En sí mismo, el concepto de liquidez nos remite a la capacidad del capital para disolver "lo sólido en el aire", tal y como cita el título de la afamada obra de Marshall Berman (1988). 
Dicho paso resulta de una importancia vital, ya que supone un gran cambio en los argumentos manageriales presentes en esta literatura, así como en los modelos de liderazgo y gestión que empiezan a ser demandados por las empresas. Se pasa de un modelo de producción en serie que presentaba una empresa jerarquizada y con alta división de tareas, a una economía constantemente cambiante con una empresa que alaba las ventajas de la ausencia de estructuras. Para esta investigación, interesa, en especial, dicho cambio reflejado en el discurso gerencial, ya que marca una nueva época en la que las funciones y la imagen del director o manager van a empezar a transformarse (Alonso y Fernández Rodríguez, 2006). Así pues, en la evolución del discurso del management, se pueden identificar tres periodos principales:

En el primero, existe una hegemonía del darwinismo social que responsabiliza al individuo de su situación en el mercado. En el segundo, la era de la gran corporación, un paradigma racional busca justificar un modelo de cooperación taylorista que supere conflictos sociales pasados. Finalmente, en el tercero encontramos la consolidación de incertidumbre total en los mercados, que demanda de empresas e individuos flexibilidad y capacidad de adaptación. (Alonso y Fernández Rodríguez, 2013, p. 59)

Se distinguen, entonces, dos grandes momentos marcados por las diferencias entre la concepción de la empresa y su gestión. Antes de comenzar, hay que tener presente que las ideologías gerenciales son producidas por medio de escuelas de gestión que nacen y se desarrollan en EE.UU, para después difundirse a lo largo del mundo, donde serán adoptadas por las escuelas europeas, latinoamericanas (Gantman, 2005, 2017) y de otros estados, como Japón. A su vez, dichos discursos también contribuyen a la conformación de las grandes potencias económicas como amenazas para la supervivencia europea, como es el caso del gigante chino en los últimos años (Fernández Rodríguez, 2011). Por tanto, cuando se habla de las modas de gestión, se refiere, en su mayoría, a las producciones norteamericanas y anglosajonas en general, modas a las que después se unirán las de otros centros económicos del mundo.

Para empezar, se encuentra un capitalismo sólido, caracterizado por la producción en serie y por una creciente intervención estatal en la economía, sobre todo durante el periodo de entreguerras. Se trata de la época en la que el sistema taylorista se convierte en el modo de producción central de las organizaciones, y el Estado del Bienestar Keynesiano redefine la organización social y el papel de las empresas en la consecución del bienestar social. En este contexto se abandona la filosofía laissez-faire, ya que se considera que el mercado no es capaz de gestionarse por sí mismo (Alonso, 2002). El Estado se vuelve intervencionista y redistributivo, al tomar conciencia del conflicto social propiciado por la economía de libre mercado. Los problemas sociales comienzan a concebirse como problemas de gestión.

Por esta razón, la literatura gerencial prescribe en este contexto "una cultura de negociación entre patronos y sindicatos, permaneciendo el conflicto social en un nivel latente" (Alonso y Fernández Rodríguez, 2006, p. 130). En su objetivo de regular la empresa desde las estructuras del Estado del Bienestar y de llegar a un acuerdo social, el discurso gerencial tendrá por objeto legitimar la posición de la dirección empresarial como la posición correcta, y marcar como incorrecta la de los sindicatos. Y es que en esta época, el discurso del management se esfuerza por desarrollarse "sobre la idea de la posibilidad de las grandes corporaciones para crear un equilibro social compatible con el máximo beneficio privado" (Alonso, 2002, p. 477), y dicha operación pasa por construir al manager como aquél que posee el poder de gestionar el conflicto.

Dicha realidad va inextricablemente unida a la constitución del sistema de relaciones laborales fordistas, que introduce la planificación y racionalización en la producción. La puesta en práctica de técnicas de racionalización y objetivación, la pasión por los datos empíricos, son una muestra clara del intento por controlar los mercados, a las que se les dota de la estabilidad necesaria para permitir su propia expansión. Esta operación se realiza mediante el management científico, que pugna por poner en valor lo racional sobre lo emocional. De este modo, no se consigue solamente controlar lo que se produce, sino también a la persona encargada de producirlo, es decir, a los/as trabajadores/as.

Para esto, se ponen en práctica técnicas de control, procedimientos de feedback, y el trabajo de supervisores y capataces que se aseguran de controlar la mejora de los procesos de producción. En este prisma, los/as managers se conciben como portadores de un saber objetivo, pero al igual que el resto de trabajadores, están 
sujetos a una disciplina del trabajo (Fernández Rodríguez, 2007). Sin embargo, su posición le otorga el poder de gestionar el conflicto desde la estructura organizacional, como ya se ha visto, pero también le convierte en portador del saber racional, hecho que a su vez determinará que sus acciones y decisiones correspondan siempre con la razón.

Llegados los años sesenta del siglo veinte, empezará a nacer una preocupación por la alienación del trabajador en la cadena de montaje taylorista, excesivamente mecanizada (Alonso y Fernández Rodríguez, 2006). La época del management científico y de la racionalización empieza a desvanecerse y se produce el surgimiento de una nueva literatura que se preocupa por la motivación del trabajador, como se puede observar en la Escuela de las Neorrelaciones Humanas. No obstante, el paso de un capitalismo sólido a otro de corte líquido no se da de forma inmediata, sino como consecuencia de crisis sucesivas, como la del petróleo del 1973, que evidencian la legitimidad del pacto keynesiano y del modelo de producción fordista.

En el mismo momento en que los modos de operar de las empresas estadounidenses dejan de ser funcionales, que coincide con un cambio en el paradigma económico mundial, Japón se dibuja como un ejemplo a imitar, ya que supone uno de los motores productivos más importantes a nivel mundial y que están a punto de desbancar el protagonismo que ostentaban hasta ese entonces los EE.UU. A diferencia de dicho país, las empresas niponas punteras ponen el acento en la gestión flexible y en la adhesión de los empleados al proyecto empresarial (Ohno, 1993), lo que logra una conciliación entre los intereses individuales y los colectivos, no de forma coercitiva, sino flexible. Debido a la progresiva fragmentación de los mercados, su internacionalización supone una reformulación de las bases estáticas sobre las que se asentaba la economía fordista.

A lo largo de los años ochenta, el sistema capitalista continúa su proceso de reestructuración, lo que permite hablar ya de un auge de la gestión flexible, la descentralización de los negocios y de la interconexión facilitada por las nuevas tecnologías. Las crisis de producción del sistema fordista, con el decrecimiento de los mercados y su incapacidad para responder a las demandas, marcará el paso de la empresa burocrática a la empresa organizada en red, logrando así dejar atrás la rigidez de las grandes corporaciones. Al mismo tiempo, los Estados-Nación ya no pueden gestionar sus mercados, pues se tornan globales, y las reglas del juego cambian. Así pues, el valor de los derechos sociales que existía en los años de entreguerras se pierde aquí para dar paso a la veneración de la competitividad global, sin anclaje, sin límites.

Entrando ya en los noventa, se puede hablar de lo que Zygmunt Bauman (2001, 2003) denomina un capitalismo líquido, basado en la concepción sobre la posmodernidad como modernidad líquida, que permite identificar el individualismo como la clave del tiempo histórico presente. En este tiempo líquido, el consumo se convierte en el eje central de las relaciones sociales, y define la exclusión social como la falta de acceso al consumo; un consumo que, según Bauman (2007), otorga a los individuos la posibilidad de construir una identidad propia en vistas de confrontar la incertidumbre, parte inextricable de la inestabilidad propia de lo líquido. Sin duda, esta concepción de la posmodernidad se relaciona con la sociedad del riesgo (Beck, 1998; Giddens, Bauman, Luhmann y Beck, 1996), ya que a mayor incertidumbre de futuro, mayor riesgo en la vida de los individuos a la hora de gestionar sus proyectos de vida.

Dentro de la modernidad líquida, los individuos deben enfrentarse a la inestabilidad, así como también las organizaciones y los estados. Ya nada es estable ni puede planificarse, tal y como ocurría en la etapa sólida, y detenerse supone la muerte. Este nuevo mundo turbulento viene dominado por las nuevas tecnologías y el discurso de las redes, hecho que influye en el flujo de capitales y la reformulación del sentido mismo del trabajo (Alonso, 1999). Como no podía ser de otro modo, la literatura gerencial hizo eco de esta situación, y pugnó por dejar atrás la alta rigidez y jerarquización que había llevado a las empresas a dicha situación crítica. Poco a poco, los mercados se iban fragmentando para dar lugar a un escenario dinámico y cambiante, donde la antigua producción en serie quedaba obsoleta para abrir paso a una economía flexible y dinámica. Por tanto, la gerencia debía amoldarse a las nuevas necesidades del mercado, y convertir el cambio en eje central de su dinámica (Alonso, 2002). 
En este sentido, la globalización económica y la deslocalización construyen un mundo repleto de incertidumbre, donde la predisposición a la acción, tanto del individuo como de la empresa, resulta esencial para prosperar. Ahora, el manager debe saber gestionar las contradicciones y sacar el máximo partido del talento y de las capacidades de su equipo desde una concepción circular y horizontal (Alonso y Fernández Rodríguez, 2006). La producción y gestión de los intangibles se convierte en el modo central de generar valor. Se habla de una etapa capitalista en la que lo simbólico es el epicentro del desarrollo económico mundial, más allá de los productos tangibles. Cabe señalar que dichas ideas, motor de la economía actual, requieren de una alta inversión en I+D+I y de un aprovechamiento total del talento de los/as trabajadores/as. La sociedad del conocimiento marca las nuevas pautas mediante la necesidad constante de generar conocimiento que fluya entre las redes del mundo. Esto tiene mucho que ver con la emergencia del discurso sobre la innovación social, que adquiere nuevos tintes en la época del capitalismo líquido.

Por esta razón, la creatividad y el arte se convertirán en valores centrales de los/as managers, ya no se realizan tareas rutinarias ni altamente fragmentadas, sino que estas deben contar con un espíritu creativo y generador de cambios. Consecuentemente, la creatividad y el arte son componentes clave de la nueva dirección empresarial que se desarrolla en el seno de la sociedad líquida y postmoderna en la que nos encontramos. En estos tiempos, "el manager postmoderno se convierte en artista, y el gerencialismo, en arte mayor" (Alonso y Fernández Rodríguez, 2006, p. 144). El control sobre los empleados ya no se desarrolla de forma unidireccional ni despótica, sino que son ellos los responsables de gestionar la inestabilidad y el cambio que caracteriza a las organizaciones, al igual que ocurre con los individuos en general, que deben encargarse de gestionar sus propios proyectos vitales en un escenario de constantes cambios y de un gran riesgo.

Así pues, la consolidación del régimen de producción posfordista trae consigo el impulso de un conjunto de valores que pasa a formar parte de los discursos sociales, como el que se refiere a la concepción del trabajador como un sujeto innovador, creador, emprendedor, visionario, etc. Se trata de un discurso que tiene por objeto convertir a los trabajadores en empresarios de sí mismos. Como se verá en el último apartado de este trabajo, las argumentaciones morales contenidas en la literatura gerencial del momento sirven para promover, no solamente entre la clase directiva, sino también entre la ciudadanía, esta lógica mercantil en la gestión de la vida de cada cual.

Dentro de esta lógica posmoderna, la innovación social pierde su sentido primigenio de adquisición de derechos sociales, y empieza a entenderse como un impulso a la sociedad del conocimiento. Al mismo tiempo, resulta central situar dicha reconceptualización de la innovación social en un contexto de financiarización y crisis del empleo (Alonso y Fernández Rodríguez, 2018); es decir, la lógica financiera pasa a formar parte de las instituciones públicas y privadas a la hora de determinar qué es lo legítimo o real. Por ejemplo, dicho concepto revela la verdadera lógica que subyace a la reciente crisis, que parece haber aumentado el poder de la economía financiera en lo social, lo que ha promovido la desregulación y la flexibilización de las relaciones laborales bajo el discurso del sacrificio (Alonso y Fernández Rodríguez, 2018).

En este contexto, en el cual se produce un descenso en las políticas sociales frente a los intereses del mercado financiero, las relaciones se basan en el sacrificio de muchos para el bienestar de unos pocos: las elites empresariales. La crisis serviría para reforzar las reglas del juego, ya que contiene una condición performativa que conforma el sentido del mundo y condiciona las subjetividades individuales. De esta manera, se desposee a la ciudadanía por medio de la producción de miedos que actúan como método disciplinario para establecer los límites de la identidad de cada cual y las posibilidades de construir un proyecto de vida de futuro con garantías. Esta lógica del nuevo gerencialismo se difunde también mediante la literatura gerencial, con lo que alcanza a la clase directiva, pero no solamente a esta, sino que también incide en la configuración de las subjetividades individuales de la población general. Esto supone que los principios de la autogestión son incorporados no solamente por directivos/as y gerentes, sino también por la ciudadanía. 


\section{Conclusión: hacia la constitución del sujeto neoliberal}

La literatura gerencial contribuye de forma decisiva en la delimitación de la ideología de la dirección empresarial, así como en su divulgación entre los cuadros directivos y el estudiantado de áreas relacionadas con los negocios. Al operar de forma articulada con otros centros de conocimiento, como las escuelas de negocios y los MBA, la literatura gerencial es uno de los pilares centrales en la divulgación de los valores proempresariales en todos los ámbitos de la sociedad. Debido a la influencia que este tipo de literatura tiene en la conformación del ethos empresarial y profesional de la clase directiva y de su extensión a gran parte de las áreas sociales, se considera de vital importancia reflexionar en torno a las argumentaciones morales que se lanzan desde este tipo de obras al grueso de la sociedad.

De forma general, se podría afirmar que la literatura gerencial del momento contribuye a la conformación de las subjetividades contemporáneas en función de los intereses del neoliberalismo. Es decir, esta literatura contribuye a la mercantilización de las capacidades de los individuos, fundamento de la expansión del capitalismo y de la legitimidad de las prácticas empresariales en la vida privada. La legitimación del nuevo espíritu del capitalismo precisa de regulaciones estatales más laxas, que permitan a las empresas operar rápidamente y cambiar de rumbo según se les vayan presentando problemas y otras situaciones adversas. Para conseguir sus objetivos, precisan que los individuos estén preparados para amoldarse a las nuevas condiciones sociales que precisan los cambios económicos, es decir que deben incorporar el discurso del riesgo (MuñozRodríguez y Santos-Ortega, 2017). Así pues, la lógica de la gestión empresarial neoliberal centrada en las características del capitalismo líquido no solamente afecta a la élite directiva, sino también a la ciudadanía, que se ve en la necesidad de gestionar sus vidas como si de una empresa se tratase.

De este modo, el discurso del riesgo cala en los individuos, quienes incorporan la idea de que si cada uno es responsable de su propio destino, la sociedad no les debe nada. Así pues, no existe un solo ámbito en el que la competencia y la libertad de elegir no sean alabadas, lo que insta a los sujetos a verse obligados a buscar la maximización de su propio interés. Convertidos en agentes racionales, los sujetos deben moverse en el ámbito de la organización laboral reconvertido en un régimen de autodisciplina que manipula las instancias psíquicas del deseo y de la culpabilización. Bajo este prisma, las problemáticas a las que se enfrentan los sujetos se justifican en su propia incapacidad para gestionar las capacidades y recursos. En este liberalismo reinventado, el sujeto actúa en una situación de mercado mediante el autogobierno, el entrepreneurship (Laval y Dardot, 2013). Todo individuo tiene algo de emprendedor en él y la economía de mercado tiene la característica de estimular dicha capacidad. Sin duda alguna, la literatura gerencial y la moda que se está divulgando en la actualidad tiene mucho que ver en la difusión de este tipo de lógica mercantil y en la vida diaria de los individuos.

De esta manera, mediante el influjo de la literatura gerencial, el sujeto aprende a gobernarse como si de una empresa se tratase. El resultado de estas operaciones se centra en la transformación de la definición del sujeto político, que deja atrás las características del sujeto productivo de la sociedad industrial. Nace un neosujeto cuya subjetividad está íntegramente implicada en la actividad que se requiere que lleve a cabo. Es decir, las nuevas técnicas de "la empresa de sí" consiguen que el individuo asuma la responsabilidad total ante los fracasos que le esperan en su camino dentro de la competitividad. Se trata de una lógica mediante la cual cada sujeto inicia el proceso de autogobernarse con base en unos valores, objetivos y principios. Esta dimensión del ethos empresarial que gobierna a los sujetos hace que se vean inmersos en un proceso de mejora de sus capacidades, como el longlife training y la empleabilidad.

En resumen, mediante el breve recorrido emprendido en este trabajo, se puede afirmar que la literatura gerencial actúa como instrumento de expansión de la lógica neoliberal entre la clase directiva y la ciudadanía, la cual incorpora gran parte de dichos valores a su actividad profesional, y que determina también el ethos empresarial en un círculo sin final que produce un sujeto cada vez más alienado y autoexplotado. De este modo, el discurso del nuevo capitalismo se convierte en un aspecto más del imaginario social, que acaba por 
trasladar el discurso de la gestión empresarial a la vida de cada individuo. Con esto, se puede finalmente hablar de la evolución de la literatura managerial como un camino marcado hacia la constitución del sujeto neoliberal más allá de la empresa.

\section{Referencias}

Abrahamson, E. (1991). Managerial Fads and Fashions: The Diffusion and Rejection of Innovations. Academy of Management Review, 16(3), 586-612. https://doi.org/10.5465/AMR.1991.4279484

Alonso, L. E. (1999). Trabajo y ciudadanía. Estudios sobre la crisis de la sociedad salarial. Madrid: Trotta.

Alonso, L. E. (2002). El discurso de la Sociedad de la Información y el declive de la reforma social. Del management del caos al caos del management. En J. García Blanco (ed.), ¿Más allá de la modernidad? Las dimensiones de la información, la comunicación y sus nuevas tecnologias (pp. 471-501). Madrid, España: Centro de Investigaciones Sociológicas.

Alonso, L. E., y Fernández Rodríguez, C. J. (2006). El imaginario managerial: el discurso de la fluidez en la sociedad económica. Política y Sociedad, 43(2), 127-151.

Alonso, L. E., y Fernández Rodríguez, C. J. (2013). Los discursos del management. Una perspectiva crítica. Lan Harremanak, 28(1), 42-69.

Alonso, L. E., y Fernández Rodríguez, C. J. (2018). Poder y sacrificio. Los nuevos discursos de la empresa. Madrid: Siglo XXI.

Álvarez, J. L., y Mazza, C. (2000). The Consumption of Management Publications. Barcelona: IESE Business School.

Bastidas, C. (2007). El caso Enron. Principales aspectos contables, de auditoria y de gobierno corporativo. Santiago de Chile: RIL Editores.

Bauman, Z. (2001). La posmodernidad y sus descontentos. Madrid: Akal.

Bauman, Z. (2003). Moderidad líquida. Buenos Aires: Fondo de Cultura Económica.

Bauman, Z. (2007). Vida de consumo. México D.F.: Fondo de Cultura Económica.

Beck, U. (1998). La sociedad del riesgo. Hacia una nueva modernidad. Barcelona: Paidós.

Berman, M. (1988). Todo lo sólido se desvanece en el aire. La experiencia de la modernidad. Madrid: Siglo XXI.

Boltanski, L., y Chiapello, È. (2002). El nuevo espiritu del capitalismo. Madrid: Akal.

Boyett, J. H., y Boyett, J. (1999). Lo mejor de los gurús: las ideas claves de Covey, Drucker, Bennis, Champy, Hammer, Kaplan, Mintzberg, Peters, Porter, Senge y los demás gurús del management. Barcelona: Gestión 2000.

Bunge, M. (2008). Filosofía y sociedad. México D.F.: Siglo XXI.

Clark, T. (2004). The Fashion of Management Fashion: A Surge Too Far? Organization, 11(2), 297-306. https://do i.org/10.1177/1350508404030659

Clark, T., y Salaman, G. (1998). Telling Tales: Management Gurus' Narratives and the Construction of Managerial Identity. Journal of Management Studies, 32(2), 137-161. https://doi.org/10.1111/1467-6486.00088

Collins, D. (2000). Management Fads and Buzzwords. Londres: Routledge.

Czarniawska, B. (1995). Narration or Science? Collapsing the Division in Organization Studies. Organization, 2(1), 11-33. https://doi.org/10.1177/135050849521002

Czarniawska, B. (1997a). A Narrative Approach to Organization Studies. Londres: Sage Publications.

Czarniawska, B. (1997b). Narrating the Organization: Dramas of Institutional Identity. Chicago: University of Chicago Press.

Engwall, L. (2012). Business Schools and Consultancies: The Blurring of Boundaries. En M. Kipping y T. Clark (eds.), The Oxford Handbook (pp. 365-388). Oxford, Reino Unido: Oxford University Press.

Fernández Rodríguez, C. J. (2007). El discurso del management: tiempo y narración. Madrid: Centro de Investigaciones Sociológicas. 
Fernández Rodríguez, C. J. (2008). Management y sociedad en la obra de Peter Drucker. RIS. Revista Internacional de Sociologia, 66(49), 195-218. https://doi.org/10.3989/ris.2008.i49.88

Fernández Rodríguez, C. J. (2011). El gato que caza nuestros ratones: China como colección de mitologías. Papeles de relaciones ecosociales y cambio global, 115, 79-88.

Fernández Rodríguez, C. J., y Medina-Vicent, M. (2017). Los nuevos discursos del management: difusión, impactos y resistencias. Recerca. Revista de pensament $i$ anàlisi, (20), 7-14. http://dx.doi.org/10.6035/Recerca.2017.20.1.

Gantman, E. (2005). Capitalism, Social Privilege and Managerial Ideologies. Aldershot, Inglaterra: Ashgate.

Gantman, E. (2017). En torno al potencial transformador de los CMS (Critical Management Studies). Recerca. Revista de pensament $i$ anàlisi, gi, 15-33. https://doi.org/10.6035/Recerca.2017.20.2

Giddens, A., Bauman, Z., Luhmann, N., y Beck, U. (1996). Las consecuencias perversas de la modernidad. Modernidad, contingencia y riesgo. Barcelona: Anthropos.

Gómez Álvarez, J., y Sánchez Barrios, M. (2009). La participación de la mujer en los consejos de administración de empresas del Ibex-35. Revista de Humanidades, 16, 105-139.

Gowler, D., y Legge, K. (1991). Images of Employees in Company Reports - Do Company Chairmen View their Most Valuable Asset as Valuable? Personnel Review, 20(3), 9-18. https://doi.org/10.1108/EUM0000000000793

Hirsch, P. M. (1972). Processing Fads and Fashions: An Organization-Set Analysis of Cultural Industry Systems. American Journal of Sociology, 77(4), 639-659. https://doi.org/10.1086/225192

Honey, P., y Mumford, A. (1982). Manual of Learning Styles. Londres: Peter Honey.

Hoopes, J. (2003). False Prophets: The Gurus Who Created Modern Management And Why Their Ideas Are Bad For Business Today. Cambridge: Basic Books.

Huczynski, A. (1993). Explaining the Succession of Management Fads. International Journal of Human Resource Management, 4(2), 444-463. https://doi.org/10.1080/09585199300000023

Huczynski, A. (2006). Management Gurus. Nueva York: Routledge.

Huczynski, A. (2011). Portrayals of Management Fashions in Contemporary Management and Organisational Behaviour Textbooks. The International Journal of Management Education, 9(3), 61-75. https://doi.org/10.37 94/ijme.93.342

Jackson, B. (2001). Management Gurus and Management Fashions. Londres/Nueva York: Taylor \& Francis.

Laval, C., y Dardot, P. (2013). La nueva razón del mundo. Ensayo sobre la sociedad neoliberal. Barcelona: Gedisa.

Mintzberg, H. (2005). Managers Not MBA's: A Hard Look at the Soft Practice of Managing and Management Development. San Francisco: Berrett-Koehler Publishers.

Muñoz-Rodríguez, D., y Santos-Ortega, A. (2017). Las cárceles del capital humano: trabajo y vidas precarias en la juventud universitaria. Recerca. Revista de pensament $i$ anàlisi, (20), 59-78. https://doi.org/10.6035/Recerca.2 017.20.4

Ohno, T. (1993). El sistema de producción Toyota: más allá de la producción a gran escala. Barcelona: Gestión 2000.

Riesman, D. (1998). On Higher Education: The Academic Enterprise in an Era of Rising Student Consumerism. Chicago: University of Chicago Press.

Sahlin-Andersson, K., y Engwall, L. (2002). The Expansion of Management Knowledge: Carriers, Flows, and Sources. Stanford: Stanford University Press.

Salmon, C. (2008). Storytelling, la máquina de fabricar historias y formatear las mentes. Barcelona: Atalaya.

Thomas, P. (2003). The Recontextualization of Management: A Discourse-based Approach to Analysing the Development of Management Thinking. Journal of Management Studies, 40(4), 775-801. https://doi.org/10.1 $111 / 1467-6486.00360$

Vail, P. B. (1991). Managing as a Performing Art: New Ideas for a World of Chaotic Change. Nueva York: Jossey-Bass.

Watson, T. J. (2004). Managers, Managism, and the Tower of Babble: Making Sense of Managerial Pseudojargon. International Journal for Sociology of Language, 166, 67-82. https://doi.org/10.1515/ijsl.2004.015 
Willmott, H. (1993). Strength Is Ignorance; Slavery Is Freedom: Managing Culture in Modern Organizations. Journal of Management Studies, 30(4), 515-552. https://doi.org/10.1111/j.1467-6486.1993.tb00315.x

\section{Notas}

* Artículo de investigación.Este artículo de investigación se inscribe en los proyectos de investigación FFI2016-76753C2-2-P?, financiado por el Ministerio de Economía y Competitividad del Gobierno de España, y UJI-A2016-04, financiado por la Universitat Jaume I.

\section{Licencia Creative Commons CC BY 4.0}

Cómo citar este artículo: Medina-Vicent, M. (2019). La evolución del discurso de la gestión empresarial a través de la literatura gerencial. Hacia la constitución del sujeto neoliberal. Signo y Pensamiento, 38(75). ht tps://doi.org/10.11144/Javeriana.syp38-75.edge 